\title{
Teses e dissertações 1 - de julho de 2020 a 30 de junho de 2021
}

\section{DOUTORADO}

ANDREUCCI JUNIOR, Sergio Jose

Identificação e análise de riscos corporativos de imagem: a relevância da Gestão de Identidades nas Relações Públicas

ORIENTADOR: Luiz Alberto Beserra de FArias

doi: Em processamento

\section{BAADER, Cinira}

Mediação comunicacional das marcas na cultura alimentar infantil

ORIENTADOR: Eneus Trindade Barreto Filho

doi: https://doi.org/10.11606/T.27.2021.tde-25082021-113248

BIERWAGEN, Gláucia Silva

Vozes da trans(formação) docente na perspectiva da Comunicação/Educação

ORIENTADOR: ADILSON ODAIR CITELLI

doi: https://doi.org/10.11606/T.27.2021.tde-31082021-195415

CARVALHO, Priscila Rezende

Entre o efêmero e as continuidades: tendências da pesquisa na interface

comunicação, moda e consumo

ORIENTADOR: EneUs Trindade Barreto Filho

doi: Em processamento

DELGADO, Flavia Daniela Pereira

Uma voz contra o autoritarismo: crônicas de Antônio Callado

durante a ditadura militar

ORIENTADORA: Sandra Lucia Amaral de Assis Reimão

doi: https://doi.org/10.11606/T.27.2020.tde-04032021-160218 
ESTEVANIM, Mayanna

Lógicas de produção jornalística em tempos de transformação digital: um pensamento sobre produto e adoção de metodologias ágeis

ORIENTADORA: Elizabeth Nicolau SAad Correa

doi: https://doi.org/10.11606/T.27.2021.tde-30082021-112856

FELICIO, Mauricio Barbosa da Cruz

Privacidade e e-vasão: uma visão propositiva sobre a privacidade em meio à aceleração comunicacional

ORIENTADORA: LUCILENE CURY

doi: https://doi.org/10.11606/T.27.2020.tde-24032021-155902

FERREIRA FILHO, Renato Gonçalves

Estética, ética e semiótica do homoerotismo pós-hiv/aids: contribuições comunicacionais e semiopsicanalíticas para a saúde pública a partir do consumo digital da pornografia amadora

ORIENTAdORA: Maria Clotilde Perez Rodrigues

doi: https://doi.org/10.11606/T.27.2021.tde-23082021-231608

FREIRE, Maria Lourdes Balbinot de Lamonica

Por que assistimos séries? Um framework do consumo de séries

ORIENTADOR: LEANDRO LEONARDo BATISTA

doi: https://doi.org/10.11606/T.27.2020.tde-02032021-131328

GARCIA, Santiago Naliato

Narrativas visuais e memória. Os acervos que constituem identidade: trabalhos fotográficos com foco no noroeste paulista ORIENTADORA: Mayra Rodrigues Gomes

doi: Em processamento

JORGE FILHO, José Ismar Petrola

Da imprensa alternativa às redes sociais: uma análise comparativa entre notícias ficcionais no Pasquim e no Sensacionalista

ORIENTADORA: Maria Cristina Castilho Costa

doi: https://doi.org/10.11606/T.27.2021.tde-24082021-214118 
MACHADO, Tariana Brocardo

Aceitação da energia nuclear por parte da opinião pública no Brasil

ORIENTADORA: Heloiza Helena Matos e Nobre

doi: https://doi.org/10.11606/T.27.2021.tde-30082021-113842

MARTINS, Juliane

Trajetórias de comunicadores desde a educação superior: histórias de vida sobre

a formação do profissional no contexto das competências

ORIENTADORA: Maria Aparecida Ferrari

doi: https://doi.org/10.11606/T.27.2021.tde-26082021-224556

PENNER, Tomaz Affonso

Bandeiras da Netflix: produção global e representações discursivas da

diversidade LGBT+ nas séries brasileiras

ORIENTAdorA: Maria Cristina Palma Mungioli

doi: Em processamento

ROSA, Beatrice Bonami

Dez categorias que expressam a interface entre tecnologia digital, comunicação

e educação: instrumento, empoderamento e rede de actantes

ORIENTADORA: BRASILINA PASSARELLI

doi: https://doi.org/10.11606/T.27.2021.tde-24082021-210027

\section{SALVATORI, Patricia Carla Gonçalves}

Ativismo em um mundo (im)perfeito: relações públicas e cidadania para

pessoas com deficiência

ORIENTADOR: Luiz Alberto Beserra de FArias

doi: https://doi.org/10.11606/T.27.2021.tde-01092021-230346

SENISE, Diego dos Santos Vega

O papel dos traços de personalidade dos indivíduos na avaliação de

propagandas

ORIENTADOR: LEANDRO LEONARDO BATISTA

doi: https://doi.org/10.11606/T.27.2021.tde-24082021-211307 
TAMURA, Natália de Campos

Entre narrativas enunciadas e o silenciamento: a interface das representações sociais de indivíduos que coabitam em um espaço urbano

ORIENTADOR: Paulo Roberto Nassar de Oliveira

doi: https://doi.org/10.11606/T.27.2020.tde-05032021-164854

\title{
MESTRADO
}

\section{ALONSO, Laiara}

Diversidade no YouTube: narrativas de Gênero, Identidade e Sexualidade pela perspectiva de Youtubers Trans

ORIENTADORA: BRASILINA PASSARELLI

doi: https://doi.org/10.11606/D.27.2020.tde-04032021-224553

\begin{abstract}
ALVES, Déborah Dias
Apelos estéticos da criação publicitária: o concretismo como tendência criativa ORIENTADOR: Victor Aquino Gomes CorreA

doi: https://doi.org/10.11606/D.27.2020.tde-04032021-144356

ARAGÃO NETO, Humberto Lima de

As transformações estéticas dos trailers cinematográficos dos anos de 1940 aos dias atuais
\end{abstract}

ORIENTADOR: Victor Aquino Gomes Correa

doi: https://doi.org/10.11606/D.27.2020.tde-01032021-145449

AZEVEDO, Ítalo Leopardi Bosco de

As representações do Samba na prática escolar como estudo de caso

ORIENTADOR: André Chaves de Melo Silva

doi: https://doi.org/10.11606/D.27.2021.tde-24082021-213544

BELONI, Patricia Oliveira

Astronomia na cobertura jornalística de ciência: representações e sociedade ORIENTADOR: André Chaves de Melo Silva

doi: https://doi.org/10.11606/D.27.2021.tde-18082021-103035 
CALDAS, Pedro Neris Luiz

Comunicação na esfera pública digital: consequências práticas do uso de Big

Data para as pesquisas de opinião pública no Brasil

ORIENTADOR: ANDERSON VINí́cIUS ROMANINI

doi: https://doi.org/10.11606/D.27.2020.tde-05032021-165215

CASTILHO, Laís Pardo

Análise discursiva de comunicação de empresas certificadas sustentáveis pelo

Sistema B-Corporation

ORIENTAdORA: Margarida Maria Krohling Kunsch

doi: Em processamento

\section{FEOLA, Gabriella Garcia Sanches}

Mapeamento da comunicação sobre sexualidade feita por influenciadores no YouTube ORIENTADORA: BRASILINA PASSARELLI

doi: https://doi.org/10.11606/D.27.2020.tde-09032021-004013

\section{FERNANDES, Kate Domingos}

Modelo de adoção de Open Banking: motivadores e barreiras que influenciam o potencial adotante

\section{ORIENTADOR: Luiz Guilherme de Carvalho Antunes}

doi: https://doi.org/10.11606/D.27.2020.tde-04032021-224111

\section{FERREIRA, Bruno Madureira}

Design de plataformas digitais: o papel da forma nas arquiteturas de participação em rede

\section{ORIENTADOR: Massimo di Felice}

doi: https://doi.org/10.11606/D.27.2021.tde-18082021-081149

\section{FRANCISCHELLI, Giovanni}

Regulação e fomento para a produção audiovisual brasileira e independente:

uma análise da política do Fundo Setorial do Audiovisual

ORIENTADOR: VÍtor SOUZA Lima BlotTA

doi: https://doi.org/10.11606/D.27.2021.tde-24082021-212752

GODOY, Eduardo Corrêa de

Memes na internet: uma análise da produção, dos usos e dos sentidos

ORIENTAdoRA: Maria Clotilde Perez Rodrigues

doi: https://doi.org/10.11606/D.27.2020.tde-26032021-153916 
GOMES, Cinthia Maria do Carmo

'O que era preto se tornou vermelho': representação, identidade e autoria negra na imprensa do século XIX por Luiz Gama

ORIENTADORA: Mayra Rodrigues Gomes

doi: https://doi.org/10.11606/D.27.2021.tde-23082021-124606

GUARDA, Rebeka Figueiredo da

O self e suas autorrepresentações nas redes sociais digitais: um estudo de perfis profissionais a partir da semiótica

ORIENTADOR: ANDERSON VinícIUS Romanini

doi: https://doi.org/10.11606/D.27.2020.tde-05032021-205841

\section{HUMES, Leonardo Bars}

Relações entre propaganda, self estendido e consumo colaborativo: uma abordagem de método misto no contexto da Airbnb ORIENTADOR: OtÁvio BANdEIRA de LAMÔNicA Freire doi: https://doi.org/10.11606/D.27.2020.tde-05032021-111647

JESUS, Thaís Cristina Afonso de Plataformas digitais de autopublicação: reflexões sobre processos criativos e editoriais ORIENTADOR: VÍtor Souza Lima Blotta doi: https://doi.org/10.11606/D.27.2020.tde-26032021-155012

KAZAN, Evelyn Medeiros

Mulheres periféricas e autorrepresentação: uma análise do Nós, Mulheres da Periferia ORIENTADORA: Claudia Lago

doi: https://doi.org/10.11606/D.27.2020.tde-09032021-002816

MAZZILLI, Bruna Sanjar

O fotolivro como espaço de complexidade e potência para a fotografia documental

ORIENTADOR: WAgner Souza E Silva

doi: https://doi.org/10.11606/D.27.2020.tde-03032021-164516 
MOTA, Cristiano Rodrigues da

Questões da Comunicação Política e da Cracolândia de São Paulo: um estudo sobre consubstancialidade, deliberação e participação cívica na imprensa

ORIENTADORA: Heloiza Helena Matos e Nobre

doi: https://doi.org/10.11606/D.27.2020.tde-04032021-143619

\section{MOURA, Cinara Martim de}

Aproximações entre comunicação, transversalidade e tecnicidades digitais: um estudo sobre a produção científica de cibercultura no Intercom

ORIENTADORA: ElizABETH NicOLAU SAAd CORREA

doi: https://doi.org/10.11606/D.27.2020.tde-08032021-230614

\section{OLIVEIRA, Emilio Alves de}

Comunicação organizacional: narrativas de empresas focadas em carreiras de jovens profissionais

ORIENTADOR: Luiz Alberto Beserra de Farias

doi: https://doi.org/10.11606/D.27.2020.tde-08032021-231939

OSHIRO, Marina Kaori Iura

League of Legends: do esporte ritual ao esporte eletrônico

ORIENTADOR: PAULO Roberto NAsSAR De Oliveira

doi: https://doi.org/10.11606/D.27.2020.tde-05032021-163646

PEREIRA, André Luis Bueno Alves

Fotografia e Identidades: expressão pessoal e representação social

ORIENTADORA: Maria Cristina Castilho Costa

doi: https://doi.org/10.11606/D.27.2020.tde-26032021-153005

ROSA, Larissa Flávia Monteiro Silva

Da manutenção à ruptura do silêncio: a cobertura jornalística da Rede Globo sobre os estupros cometidos pelo médium 'João de Deus'

ORIENTADORA: MAyra Rodrigues Gomes

doi: https://doi.org/10.11606/D.27.2021.tde-18082021-095447 
SENLLE, Renata Garcia

Conversas de Mães em Rede: um percurso netnográfico, dos blogs maternos até a Bancada de Mães Ativistas nas eleições de 2018 no Facebook

ORIENTADOR: Paulo Roberto Nassar de Oliveira

doi: https://doi.org/10.11606/D.27.2020.tde-05032021-210528

SERRALHEIRO, Vinícius Alves

Existe amor em app?: Percepções sobre a sexualidade, a prevenção e a comunicação do HIV e da aids entre usuários de aplicativos de relacionamento ORIENTADOR: LEANDRO LEONARDO BATISTA

doi: https://doi.org/10.11606/D.27.2020.tde-27032021-120511

SOUZA, Gabriel Moni de

O futebol brasileiro mediado e midiatizado: uma análise semiótica da marca Corinthians e suas expressividades

ORIENTADORA: Maria Clotilde Perez Rodrigues

doi: Em processamento

SOUZA, Manuela Thamani Nascimento de

Futuro se faz com a história, e história com o povo dentro: Movimentos Negros na interface da Comunicação e Educação

ORIENTADORA: Claudia LAgo

doi: https://doi.org/10.11606/D.27.2021.tde-18082021-101653

TAVARES, Mariane Beline

O contemporâneo hiperconectado: realidade aumentada e seus usos nas redes sociais e nos videogames

ORIENTADORA: BRASILINA PASSARELLI

doi: https://doi.org/10.11606/D.27.2020.tde-05032021-120846

VELLEI, Carolina dos Santos

Os Stories jornalísticos no Instagram: investigando os novos formatos de narrativas nas mídias sociais

ORIENTADOR: WAgner Souza E Silva

doi: https://doi.org/10.11606/D.27.2020.tde-08032021-230133 